\title{
Gênero e manifestação de stress em hipertensos
}

\author{
Gender and the manifestation of stress \\ in patients hypertensive
}

\author{
Shana Hastenpflug WOTTRICH \\ Camila de Matos ÁVILA² \\ Clenise Canello MACHADO \\ Silvia GOLDMEIER ${ }^{3}$ \\ Denise DILLENBURG $^{4}$ \\ Cristiana Palma KUHL ${ }^{5}$ \\ Maria Cláudia IRIGOYEN ${ }^{6}$ \\ Katya RIGATTO \\ Patrícia Pereira RUSCHEL ${ }^{3}$
}

\begin{abstract}
Resumo
Embora estudos sustentem maior vulnerabilidade ao stress na população feminina, há escassez de dados sobre a população de hipertensos. Realizou-se um estudo transversal para verificar a diferença na prevalência de sintomas de stress entre os gêneros, por meio da aplicação do Inventário de Sintomas de Stress de Lipp e de um questionário de dados sociodemográficos. Os resultados não mostram diferença estatisticamente significativa concernente à presença de sintomas de stress entre as amostras masculina ( $n=33$ ) e feminina ( $n=70$ ). Contudo, mais homens apresentaram escores na fase de resistência $(60,6 \%)$, enquanto a fase de quase-exaustão preponderou entre as mulheres: 41,4\% versus 15,2\% dos homens. Considerando sintomas físicos e psicológicos no geral, independente de predominância de fase, as mulheres relataram ter significativamente mais sintomas psicológicos de stress nos escores referentes às fases de resistência, quase-exaustão e à fase de exaustão $(p<0,05)$. Esses achados podem auxiliar no planejamento de abordagens de controle de stress.
\end{abstract}

Unitermos: Estresse. Gênero. Hipertensão.

\begin{abstract}
Even though studies support the idea of greater vulnerability to stress in the female population, there is a dearth of studies related to hypertensive patients. A cross-sectional study was conducted with the aim of verifying the difference in the prevalence of stress

$\boldsymbol{\nabla \nabla \nabla \nabla}$

1 Universidade Federal de Santa Maria, Programa de Pós-Graduação em Mestrado de Psicologia. Porto Alegre, RS, Brasil.

2 Psicóloga. Porto Alegre, RS, Brasil.

3 Fundação Universitária de Cardiologia, Instituto de Cardiologia, Serviço de Psicologia Clínica. Av. Princesa Isabel, 395, Bairro Santana, 90620-001, Porto Alegre, RS, Brasil. Correspondência para/Correspondence to: P.P. RUSCHEL. E-mail: <psicologia.clinica@cardiologia.org.br>.

- Fundação Universitária de Cardiologia, Instituto de Cardiologia, Serviço de Nutrição e Dietética. Porto Alegre, RS, Brasil.

5 Fisoterapeuta hospitalar. Porto Alegre, RS, Brasil.

- Universidade São Paulo, Instituto do Coração. São Paulo, SP, Brasil.

Agradecimentos: À psicóloga Josiane Lieberknecht Wathier Abaid pela leitura cuidadosa e crítica, que muito contribuiu para a finalização desse texto.
\end{abstract}


symptoms in a male and female sample of hypertensive patients, using Lipp's Adult Stress Symptoms Inventory and a socio-demographic questionnaire. There was no statistically significant difference in respect of the presence of stress symptoms between males $(n=33)$ and females $(n=70)$. However, men obtained higher scores in the resistance phase (60.6\%), while women obtained higher scores in the near-exhaustion phase (41.4\% versus $15.2 \%$ of men). Considering overall physical and psychological symptoms, irrespective of the predominant phase, women reported significantly higher psychological stress symptoms than men in terms of the scores in the resistance and near-exhaustion phases, as well as the scores in the exhaustion phase $(p<0.05)$. These findings may help in the planning of stress-management strategies.

Uniterms: Stress. Gender. Hypertension.

As doenças cardiovasculares são as principais causas de morte no Brasil. Em 2004, a taxa de mortalidade específica (por 100 mil habitantes) por doenças isquêmicas do coração no Brasil atingiu a marca de 47,80 (Ministério da Saúde, 2006), mostrando a necessidade de consideração desse problema nas políticas de saúde pública.

A hipertensão arterial é um dos principais fatores de risco para o desenvolvimento das doenças cardiovasculares; sua presença está relacionada a altas taxas de morbimortalidade relacionada a doenças cardíacas (Sociedade Brasileira de Cardiologia - SBC, 1998; 2006). É conceituada como uma situação clínica caracterizada por elevação nos níveis tensionais da parede de vasos sanguíneos (pressão arterial sistêmica), e também como uma doença crônico-degenerativa, que compromete fundamentalmente o equilíbrio dos sistemas vasodilatadores e vasoconstrictores, ocasionando a redução da luz dos vasos e danos aos órgãos por eles irrigados. É concebida como uma síndrome de múltiplas etiologias, podendo ser associada a alterações metabólicas e hormonais (Irigoyen, Krieger \& Consolim-Colombo, 2005; Ribeiro, 1996; Swales, 1994; SBC, 2006).

Sua prevalência na população brasileira é elevada. Segundo Lessa (2001), estima-se que atinja cerca de 25\% da população. Furtado e Martin (1995) referem que as mulheres têm maior proteção hormonal que os homens contra eventos cardiovasculares até a menopausa. Uma associação de fatores - desde alterações comportamentais, como tabagismo e obesidade, até questões relacionadas à diminuição da produção de estrogênio na mulher - constitui risco maior para doenças cardiovasculares na fase que segue a menopausa (Gorodeski, 2002). O stress é mencionado como possível fator modificador da atividade neuroendócrina simpática e das respostas cardiovasculares a estímulos. Diante dessas colocações, pode-se pensar em uma diversidade de fatores físicos, de ordem psicológica e comportamental, que quando associados aumentam o risco de doenças cardiovasculares como a hipertensão.

Dentre os fatores psicológicos, a literatura aborda traços de personalidade característicos do indivíduo hipertenso. A investigação do funcionamento psicodinâmico desses sujeitos revela a existência de impulsos agressivos inibidos, concomitantes à presença de ansiedade. Também se destacam características relacionadas à inabilidade na expressão de impulsos agressivos e sentimentos, inassertividade - isto é, falta de habilidade para sustentar opiniões ou posicionamentos e expressar afetos -, esforço para manutenção de um grau de controle, senso de responsabilidade exagerado, preocupação com metas, condescendência, submissão e necessidade de se mostrar agradável aos outros (Gandarillas, Câmara \& Scarparo, 2005; Lipp, 1995; Lipp \& Rocha, 1994, 2007; Maciel, 2002).

Dentro do consenso e das diretrizes nacionais elaboradas acerca da hipertensão arterial sistêmica, o stress é abordado com um dos fatores de risco cardiovascular para aqueles pacientes com pressão limítrofe (SBC, 1998; 2006). Estudos evidenciam o efeito do stress na reatividade cardiovascular e na pressão arterial, sugerindo que poderia contribuir para a hipertensão arterial sustentada (Lipp, Pereira, Justo \& Matos, 2006; SBC, 2006). Para compreender a interação entre fatores psicológicos e a reatividade cardiovascular em momentos de stress, estudo de Lipp, Frare e Santos (2007) identificou correlação significativa entre alexitimia (dificuldade de identificar e de expressar emoções) e inassertividade e reatividade da pressão arterial. Tais resultados corroboram a intersecção entre os fatores psicológicos descritos e a reatividade cardiovascular, apontando a importância de intervenções psicológicas para redução de stress em programas de tratamento para hipertensos.

O stress é definido como uma reação do organismo que se dá de forma complexa e global, envol- 
vendo componentes físicos, psicológicos, mentais e hormonais. É um estado de tensão que causa ruptura no equilíbrio interno do organismo, gerada quando há necessidade de atender demandas que superem a capacidade adaptativa do indivíduo (Lipp, 2000; Lipp \& Rocha, 1994; Selye, 1965). Em outras palavras, o stress é desencadeado como reação ao enfrentamento de situações que amedrontem, ameacem ou desafiem o indivíduo (Lipp \& Rocha, 2007). Desenvolve-se em quatro fases: alerta, resistência, quase-exaustão e exaustão (Lipp, 2000). Nas duas últimas fases, instala-se o processo de adoecimento, ou seja, aqueles órgãos com maior vulnerabilidade genética ou adquirida mostram sinais de deterioração e adoecem. Assim sendo, quando o stress é prolongado, é possível que afete o sistema imunológico do indivíduo, tornando-o vulnerável a doenças (Lipp \& Rocha, 2007).

Do ponto de vista da prevalência do stress na população brasileira, a questão do gênero é ainda um ponto controverso. Calais, Andrade e Lipp (2003) sustentam maior vulnerabilidade ao stress na população feminina, focando-se em adultos jovens. Porém, ressaltam a especificidade de diferenças sexuais, dependendo do estressor e da desordem envolvidos. Concordam com essa estatística os índices de stress encontrados na população de magistrados da Justiça do Trabalho (Lipp \&Tanganelli, 2002).

Fatores que influenciam a relação entre nível de stress e gênero feminino podem estar subjacentes a mudanças no papel social das mulheres na sociedade moderna (Aquino, Menezes, Amoedo \& Nobre, 1991; Straub, 2005). Segundo Aquino et al. (1991), as condições sociais exigem das mulheres um esforço adaptativo maior para lidar com as exigências da vida diária - tanto no ambiente doméstico, quanto no de trabalho -, causando um aumento no nível de stress. Lundberg e Frankenhaeuser (1999) atestam que os níveis de stress nas mulheres tendem a continuar elevados após o trabalho, diante das atividades domésticas, enquanto o dos homens tende a diminuir após o fim do expediente. Dados sustentam também que o nível de stress despertado por situações específicas é determinado pela forma como o indivíduo compreende, interpreta e sente-se a respeito dessas situações (Moreira \& Mello Filho, 1992; Straub, 2005).

Dados de pesquisas mostram que mulheres buscam mais precocemente tratamentos de saúde. Os homens padecem mais de condições severas e crônicas e são menos presentes em serviços de atenção primária do que as mulheres. Considera-se que o fato de se sentirem invulneráveis faça com que se exponham mais e acabem ficando vulneráveis (Gomes, Nascimento \& Araújo, 2007; Straub, 2005). Caberia considerar, assim, padrões de interpretação e enfrentamento gênero-dependentes de situações, o que explicaria a relação entre níveis mais altos de stress e o gênero feminino.

Embora a relação entre hipertensão arterial sistêmica e stress seja ainda discutida na literatura, poucos estudos mostram a relação entre stress e gênero na população de hipertensos. Assim, o objetivo deste estudo é verificar a diferença na prevalência de sintomas de stress na população feminina e na masculina de pacientes hipertensos atendidos no ambulatório multiprofissional de hipertensão arterial sistêmica em um centro de referência cardiológica do Rio Grande do Sul.

\section{Método}

\section{Participantes}

Cento e três adultos (70 mulheres e 33 homens) foram entrevistados por psicólogos treinados para aplicação do instrumento da pesquisa, sendo abordados sequencialmente na medida em que eram atendidos pela equipe de saúde. Foram incluídos no estudo pacientes maiores de 18 anos, previamente agendados para as consultas ambulatoriais para tratamento de hipertensão, de junho a novembro de 2007, em centro de referência cardiológica no Rio Grande do Sul. Como critérios de exclusão foram considerados ter idade inferior a 18 anos, ser analfabeto ou não ter condições físicas ou psicológicas/psiquiátricas para responder ao instrumento. Todos os pacientes entrevistados estavam em uso de medicação anti-hipertensiva. A escolha pelo tamanho da amostra foi baseada em pesquisas anteriores (Calais et al., 2003; Lipp \& Tanganelli, 2002), para que fosse possível detectar uma diferença absoluta da presença de stress de aproximadamente $26 \%$, considerando poder de $80 \%$ e $\mathrm{p}<0,05$.

\section{Instrumentos}

Para coleta dos dados foram utilizados: uma ficha de dados sociodemográficos e o Inventário de Sintomas de Stress para Adultos de Lipp (ISSL) (Lipp, 2000). 
Os itens avaliados na ficha de dados sociodemográficos foram idade, gênero, estado civil, escolaridade, atividade laboral, tempo de conhecimento da doença e tempo de tratamento medicamentoso para a doença.

O ISSL (Lipp, 2000) é um instrumento que visa identificar de modo objetivo os sintomas de stress apresentados pelo paciente, avaliando o tipo de sintomas presentes (somáticos ou psicológicos) e a fase de stress em que o paciente se encontra. É composto de três quadros, constituídos por um total de 37 itens, que reportam sintomas de stress de natureza somática e 19 itens de natureza psicológica. $O$ instrumento é respondido a partir do assinalamento dos sintomas presentes nas últimas 24 horas, na última semana e no último mês .

O banco de dados foi digitado em planilha eletrônica Microsoft Excel e analisado pelo software estatístico Statistical Package for the Social Sciences (SPSS) versão 15.0 for Windows. A análise descritiva foi realizada por meio da Média (M), Desvio-Padrão (DP) ou mediana (Percentis 25 e 75) para as variáveis contínuas; e frequências absolutas e relativas para as categóricas. Para verificar a associação de gênero com a presença de stress foi utilizado o teste qui-quadrado de Pearson. Para verificar a diferença entre a presença de sintomas físicos e psicológicos de stress entre os gêneros, considerando as médias, independentemente da predominância de fase, foi utilizado teste $t$ de Student. Foram considerados significativos os resultados com $p<0,05$.

Mediante aprovação do projeto de pesquisa que deu origem a esse estudo pelo Comitê de Ética em Pesquisa da instituição, iniciou-se a coleta dos dados. Os participantes foram abordados em consultas de rotina do ambulatório com o psicólogo, sendo convidados a participar da pesquisa voluntariamente, e esclarecidos sobre o tema do estudo e suas implicações, bem como sobre seus direitos como sujeitos de pesquisa. Receberam antes da execução das entrevistas um Termo de Consentimento Livre e Esclarecido, que autoriza sua participação no estudo a ser realizado.

Foram seguidos os preceitos da Resolução de 196/96 do Conselho Nacional de Saúde (Conselho Nacional de Saúde, 1997) e a Resolução 016/2000 do Conselho Federal de Psicologia (Conselho Federal de Psicologia, 2000), que normatizam as condições da pes- quisa que envolvem seres humanos, considerando a ética, a preservação da identidade dos participantes e não lhes oferecendo riscos ou perdas.

\section{Resultados}

Inicialmente, apresentam-se as medidas pressóricas dos pacientes. Posteriormente, são explicitadas as análises descritivas com o perfil sociodemográfico dos participantes do estudo conforme o gênero a que pertencem (Tabela 1). Em seguida, são expostos os dados que verificaram diferenças por gênero quanto ao instrumento ISSL, assim como a especificação por fase de stress detectada nos participantes.

As medidas da pressão arterial sistólica (PAS) do grupo de pacientes avaliados variaram entre $240 \mathrm{mmHg}$ e 114mmHg, com média de 167, DP=33mmHg e pressão arterial diastólica (PAD) entre $160 \mathrm{mmHg}$ e $60 \mathrm{mmHg}$, com média 97, DP=19mmHg. Especificamente, as medidas das pressões dos homens tiveram uma média de PAS 166, DP=33 mmHg e PAD 97, DP=19mmHg e as medidas de pressão das mulheres tiveram média de PAS 167, DP=33mmHg e PAD 97, DP=19mmHg.

Dentre os dados apresentados, destaca-se uma tendência estatística $\left(\boldsymbol{\chi}^{2}=2,96 ; \mathrm{gl}=1 ; p=0,08\right)$ : uma percentagem maior de homens que vivem com uma companheira do que de mulheres com companheiro. Não houve diferença estatística quanto à escolaridade ou quanto à atividade laboral por gênero. No entanto, há diferença estatisticamente significativa quanto ao tratamento medicamentoso para a hipertensão: as mulheres tratam-se há mais tempo do que os homens (mediana de 46,76 meses das mulheres versus 29,50 meses dos homens) $(U=412,50 ; z=-3,032 ; p=0,002)$.

Não houve diferença significativa em relação à presença ou ausência de sintomas de stress quando comparadas as amostras masculina e feminina $(p=0,078)$. Dos homens, 84,8\% tiveram escores compatíveis com a presença de stress versus 85,7\% das mulheres.

Dos 103 entrevistados, 88 apresentaram sintomas compatíveis com a presença de stress (Tabela 2). Os resultados apontam a predominância de sintomas referentes à fase de resistência, quando considerados todos os sujeitos da amostra. Considerando o gênero, encontrou-se uma percentagem maior de homens na fase de resistência $(60,6 \%)$, enquanto a fase de quase- 
-exaustão preponderou entre as mulheres $(41,4 \%$ contra $15,2 \%$ dos homens).

Na fase de resistência, nos escores de nove homens, predominaram os sintomas físicos, e de outros nove, os sintomas psicológicos, além de dois terem predominância de sintomas mistos. Com relação às mulheres, 19 tiveram predominância de sintomas psicológicos, sete de físicos e dois de mistos nessa fase. $\mathrm{Na}$ fase de quase exaustão, para 19 mulheres predominaram os sintomas psicológicos, para seis, os sintomas físicos, e para quatro, os sintomas mistos. Poucos homens tiveram escores compatíveis com stress nessa fase. Ambos

Tabela 1. Características sociodemográficas e gênero. 2007.

\begin{tabular}{|c|c|c|c|c|}
\hline \multirow{3}{*}{$\begin{array}{l}\text { Características sociodemográficas } \\
\text { Idade } \\
\text { Estado civil }\end{array}$} & \multirow{2}{*}{\multicolumn{2}{|c|}{$\begin{array}{c}\text { Homens }(n=33,32 \%) \\
M=47,91, D P=14,56\end{array}$}} & \multirow{2}{*}{\multicolumn{2}{|c|}{$\begin{array}{c}\text { Mulheres }(\mathrm{n}=70,68 \%) \\
\mathrm{M}=51,76, \mathrm{DP}=11,86\end{array}$}} \\
\hline & & & & \\
\hline & $\mathrm{n}$ & $\%$ & $n$ & $\%$ \\
\hline Com companheiro(a) & 27 & 81,8 & 45 & 64,3 \\
\hline Sem companheiro(a) & 6 & 18,2 & 24 & 34,3 \\
\hline Não responderam & 0 & 0 & 1 & 1,43 \\
\hline Escolaridade & $\mathrm{n}$ & $\%$ & $n$ & $\%$ \\
\hline$<8$ anos & 18 & 54,5 & 42 & 60,0 \\
\hline$\geq 8$ anos & 14 & 42,4 & 26 & 37,1 \\
\hline Não responderam & 1 & 3,0 & 2 & 2,8 \\
\hline Atividade laboral & $\mathrm{n}$ & $\%$ & $n$ & $\%$ \\
\hline Ativos & 14 & 42,4 & 25 & 35,7 \\
\hline Inativos & 17 & 51,5 & 26 & 37,1 \\
\hline Não responderam & 2 & 6,0 & 19 & 27,1 \\
\hline Tempo de conhecimento da doença - mediana em meses $\left(P_{25} ; P_{75}\right)$ & 108 & $45 ; 162$ & 180 & $96 ; 282$ \\
\hline Tempo de tratamento medicamentoso - mediana em meses $\left(\mathrm{P}_{25^{\prime}} \mathrm{P}_{75}\right)$ & 29,50 & $39 ; 150$ & 46,76 & $60 ; 240$ \\
\hline
\end{tabular}

M: média; DP: desvio-padrão.

Tabela 2. Distribuição das fases de stress quanto ao gênero. 2007.

\begin{tabular}{llllccccc}
\hline & \multicolumn{2}{l}{ Fase de alerta } & \multicolumn{2}{l}{ Fase de resistência } & Fase de quase-exaustão & \multicolumn{2}{c}{ Fase de exaustão } \\
\hline & $\mathrm{n}$ & $\%$ & $\mathrm{n}$ & $\%$ & $\mathrm{n}$ & $\%$ & $\mathrm{n}$ & $\%$ \\
Homens & 2 & 6,1 & 20 & 60,6 & 5 & 15,2 & 1 & 3,0 \\
Mulheres & 1 & 1,4 & 28 & 40,0 & 29 & 41,4 & 2 & 2,9 \\
\hline Total & 3 & 2,9 & 48 & 46,6 & 34 & 33,0 & 3 & 2,9 \\
\hline
\end{tabular}

Tabela 3. Frequência de sintomas gerais de stress, independentemente da predominância de fase, quanto ao gênero. 2007.

\begin{tabular}{|c|c|c|c|c|c|}
\hline & \multicolumn{2}{|c|}{ Homens } & \multicolumn{2}{|c|}{ Mulheres } & \multirow{2}{*}{$p$} \\
\hline & $n=33$ & $32 \%$ & $n=70$ & $68 \%$ & \\
\hline \multicolumn{6}{|l|}{ Fase de alerta } \\
\hline Sintomas físicos & 40,36 & 26,30 & 42,86 & 22,63 & 0,622 \\
\hline Sintomas psicológicos & 32,12 & 32,72 & 27,43 & 31,96 & 0,492 \\
\hline Fase de resistência e fase de quase-exaustão & $n=33$ & $32 \%$ & $n=70$ & $68 \%$ & \\
\hline Sintomas físicos & 42,73 & 22,95 & 50,71 & 27,31 & 0,149 \\
\hline Sintomas psicológicos & 43,03 & 32,83 & 63,43 & 31,75 & $0,003^{*}$ \\
\hline Fase de exaustão & $n=33$ & $32 \%$ & $\mathrm{n}=70$ & $68 \%$ & \\
\hline Sintomas físicos & 32,75 & 19,13 & 34,65 & 19,42 & 0,643 \\
\hline Sintomas psicológicos & 38,21 & 27,72 & 54,46 & 31,93 & $0,014^{*}$ \\
\hline
\end{tabular}

${ }^{*}$ Valores estatisticamente significantes. 
os gêneros não acusaram predomínio de sintomas físicos e mistos na fase de exaustão.

Quando considerados os sintomas no geral, independentemente de predominância de fase, houve diferença significativa entre homens e mulheres referentes aos tipos de sintomas predominantes (físicos e psicológicos) nos quadros da testagem que dizem respeito às fases mais avançadas de stress (resistência, quase-exaustão e exaustão). Conforme descrito na Tabela 3, as mulheres relataram ter significativamente mais sintomas psicológicos de stress do que os homens nos escores referentes às fases de resistência e quase-exaustão e nos escores referentes à fase de exaustão $(p<0,05)$.

\section{Discussão}

Diante da amostra de sujeitos predominantemente feminina, pode-se considerar a ideia de que mais mulheres do que homens buscam assistência à saúde nesse ambulatório. Além disso, percebe-se que a mediana do tempo de tratamento medicamentoso para a hipertensão difere para os entrevistados homens e mulheres. As mulheres da amostra parecem ter buscado tratamento mais precocemente, concordando com outros estudos (Straub, 2005). Segundo Gomes, Nascimento e Araújo (2007), a dificuldade de os homens buscarem os serviços de saúde estaria vinculada ao imaginário social, à ideia de que o homem é invulnerável a doenças. Esse dado pode estar relacionado à condição conjugal dos homens da amostra, que, em sua maioria, vivem com uma companheira, isto é, aqueles que buscam atendimento são de alguma forma influenciados pela companheira, sendo possível pensar no casamento como um fator de proteção para doenças (Schraiber, Gomes \& Couto, 2005).

Os dados evidenciam que a escolaridade dos entrevistados é baixa e que praticamente metade dos sujeitos da amostra não tem nenhuma atividade laboral; devido a isso, não houve diferença estatisticamente significativa por gênero nessas variáveis. Essa informação remete ao perfil dos pacientes assistidos pela rede de serviços do SUS, que em sua maioria estão em condição social precária. Para Romano (2001), o baixo nível de educação está relacionado à forma como os pacientes percebem e enfrentam a doença. O status socioeconô32 mico, em certa medida, determina a maneira como os indivíduos lidam com as situações estressantes em suas vidas, acessam as fontes de suporte social e organizam-se psiquicamente para enfrentar as adversidades: fatores importantes no que diz respeito à aderência ao tratamento.

Ao contrário de outros estudos (Calais et al., 2003; Lipp \& Tanganelli, 2002), os resultados não apontam diferença significativa quanto à presença de stress entre os gêneros. Há considerável discussão na literatura, que pontua existir uma mudança de paradigmas vigentes na cultura brasileira. Inicialmente, pensava-se que os homens teriam maior nível de stress do que as mulheres, pois seus papéis sociais de mantenedores da família gerariam sobrecarga afetiva e intelectual (Calais et al., 2003). Mais recentemente, tem-se discutido sobre a mudança da função da mulher no universo doméstico e profissional. Aquino et al. (1991) e Lundberg e Frankenhaeuser (1999) sugerem que o nível de stress feminino vem aumentando em decorrência da maior participação das mulheres no mercado de trabalho. Assim, haveria a soma das exigências profissionais com as atribuições domésticas, o que explicaria os níveis mais elevados de stress nas mulheres quando comparadas aos homens. Como os resultados dessa pesquisa fogem desse padrão, pode-se pensar na existência de particularidades na população de hipertensos.

Sendo a hipertensão uma doença crônica e considerando-se o stress um dos fatores etiológicos da doença, é possível sugerir que homens e mulheres hipertensos mantêm níveis de stress mais elevados do que os do restante da população, o que explicaria a ausência de diferença significativa entre os gêneros. Ao mesmo tempo, a própria situação de adoecimento pode ser pensada como geradora de feedback negativo sobre o stress. Se o stress causa a hipertensão, as condições limitantes e frustrações promovidas pela situação de doença podem incrementar também os sintomas de stress, gerando um ciclo vicioso que pode culminar no agravamento do quadro clínico (Romano, 2001). Surge daí a necessidade de se atentar para esse fator de risco, a fim de se instituírem estratégias de manejo e enfrentamento do stress para essa população.

Estudo realizado com adultos jovens (Calais et al., 2003) e estudo com Magistrados da Justiça do Trabalho (Lipp \& Tanganelli, 2002) mostraram que a maioria dos participantes das pesquisas estava na fase de 
resistência. Esses resultados concordam com os encontrados nesta pesquisa: 46,6\% do total dos sujeitos da amostra encontram-se nessa fase de stress. A fase de resistência é caracterizada por dificuldades com a memória, por cansaço físico e mental, bem como pela ampliação da vulnerabilidade a doenças causadas por vírus e bactéria, além daquelas que têm como pressuposto a predisposição genética (Lipp, 1995 ).

Embora a diferença não seja estatisticamente significativa, considerando o gênero, os homens mostraram mais escores na fase de resistência (60,6\%), enquanto para as mulheres predominou a fase de quase-exaustão $(41,4 \%)$, seguida da fase de resistência (40,0\%). Chamam a atenção os escores de homens e mulheres quando comparados na fase de quase-exaustão, pois há diferença na predominância de stress nesse nível: 15,5\% dos homens versus 41,4\% das mulheres.

Essa fase é caracterizada pelo começo do colapso do organismo e pelo início do seu adoecimento (Lipp, 2000; Silva, 2005). Dessa forma, doenças com predisposição genética, tais como a hipertensão, são facilitadas pela vulnerabilidade causada pelo stress crônico (Silva, 2005). Pode-se considerar que as mulheres hipertensas desse ambulatório demonstram mais sintomas crônicos do que os homens. Nesse sentido, cabe questionar se o fato de homens e mulheres apresentarem sintomas mais ou menos intensos de stress não estaria ligado aos seus sistemas de crenças, percepções e formas de enfrentamento das adversidades, incluindo a situação de doença (Lipp \& Rocha, 1994; Moreira \& Mello Filho, 1992; Silva, 2005; Straub, 2005). A partir desses resultados, surge a hipótese de que o nível de stress nessa amostra seja maior do que o encontrado na população em geral, o que dispersaria a diferença entre os gêneros.

A diferença estatisticamente significativa entre homens e mulheres no que concerne à referência de sintomas psicológicos nos escores das fases mais avançadas de stress evidencia maior vulnerabilidade das mulheres na esfera psicológica. Esse resultado aponta para a necessidade de implantação de estratégias de enfrentamento do stress que considerem a vulnerabilidade de cada gênero.

Esse estudo foi realizado em amostra de pacientes de um único ambulatório, em uma região específica do país. Portanto, os resultados não podem ser generalizados para a população brasileira. Além disso, por serem baseados na aplicação de um instrumento de autorrelato, os resultados retratam a perspectiva dos participantes sobre seus sintomas de stress. Para diminuir tal viés, sugerimos a triangulação de dados, inserindo a aplicação de instrumentos que meçam a percepção de stress a partir da perspectiva de familiares ou de pessoas próximas aos pacientes. Sugere-se, portanto, que o escopo da pesquisa seja ampliado para outras populações de hipertensos a fim de aumentar o alcance dos resultados aqui obtidos.

\section{Considerações Finais}

O presente trabalho revela que não há diferença significativa de stress entre os gêneros na população de hipertensos atendidos em ambulatório multiprofissional de hipertensão arterial sistêmica de um centro de referência cardiológica do Rio Grande do Sul. O estudo aponta diferença, embora não estatisticamente significativa, quanto às fases de stress, destacando que há mais mulheres com sintomas em fase avançada de stress do que homens. É importante destacar que nas usuárias desse serviço, o stress parece acontecer de forma mais crônica. Os dados levam a questionar se a própria situação de doença não torna as mulheres mais vulneráveis ao stress, e indicam existência de diferença significativa quanto aos sintomas psicológicos, que são predominantes nas mulheres, destacando uma possível predisposição psicológica dessa população aos sintomas de stress em fases avançadas.

Considerando que as V Diretrizes de Hipertensão Arterial preconizam o uso de treinamento para controle do stress como medida adicional na abordagem não farmacológica de pacientes hipertensos, conhecer o paciente pode representar uma importante ferramenta para planejar as abordagens de controle de stress específicas para essa população. Tal conduta pode ser especialmente importante se levarmos em conta as diferenças nos sintomas apresentados por cada gênero.

\section{Referências}

Aquino, E. M. L., Menezes, G. M., Amoedo, M. B. E, \& Nobre, L. C. C. (1991). Mortalidade feminina no Brasil: gênero frágil ou gênero forte? Cadernos de Saúde Pública, 7 (2). Recuperado em 2007 janeiro 8, 2007, disponível em http://www.scielo.br/ pdf/csp/v7n2/v7n2a04.pdf 
Brasil. Ministério da Saúde. (2006). Datasus: indicadores e dados básicos. Recuperado em janeiro 29, 2008, disponível em http://tabnet.datasus.gov.br/cgi/deftohtm.exe? idb2006/c08.def

Calais, S. L., Andrade, L. M. B., \& Lipp, M. E. N. (2003). Diferenças de gênero e escolaridade na manifestação de stress em adultos jovens. Psicologia: Reflexão \& Crítica, 16 (2), Recuperado em janeiro 8, 2007, disponível em http:// www.scielo. br/pdf/prc/v16n2/a05v16n2.pdf

Conselho Federal de Psicologia. (2000). Resolução para pesquisas com seres humanos: Res. 016/2000. Brasília: Autor.

Conselho Nacional de Saúde. (1997). Resolução para pesquisas envolvendo seres humanos: Res. 196/1996. Brasília: Autor.

Furtado, M. R., \& Martin, J. F. (1995). Hipertensão arterial na mulher: um caso especial? Arquivos Brasileiros de Cardiologia, 64 (2), 141-144.

Gandarillas, M. A., Câmara, S. G., \& Scarparo, H. (2005). Estressores sociais da hipertensão em comunidades carentes. Psicologia: Reflexão \& Crítica, 18 (1). Recuperado em janeiro 8, 2007, disponível em http://www.scielo.br/ $\mathrm{pdf} / \mathrm{prc} / \mathrm{v} 18 \mathrm{n1} 1 / 24818 . \mathrm{pdf}$

Gomes, R., Nascimento, E. F., \& Araújo, F. C. (2007). Por que os homens buscam menos os serviços de saúde do que as mulheres? As explicações de homens com baixa escolaridade e homens com ensino superior. Cadernos de Saúde Pública, 23 (3). Recuperado em outubro 12, 2008, disponível em http://www.scielo.br/pdf/csp/v23n3/ 15.pdf

Gorodeski, G. I. (2002). Update on cardiovascular disease on post-menopausal women. Best Practice and Research Clinical Obstetrics Gynaecology, 16 (3). Retrieved, May 28, 2007 available from http://www.sciencedirect.com/ science

Irigoyen, M. C., \& Krieger, E. M., Consolim-Colombo, F. M. (2005). Controle cardiovascular: regulação reflexa e papel do sistema nervoso simpático. Revista da Sociedade Brasileira de Hipertensão, 8 (1), 6-10.

Lessa, I. (2001). Epidemiologia da hipertensão arterial sistêmica e da insuficiência cardíaca no Brasil. Revista Brasileira de Hipertensão, 8 (4). Recuperado em outubro 11, 2008, disponível em http://departamentos.cardiol. br/dha/ revista/8-4/epidemiologia.pdf

Lipp, M. E. N. (1995). Stress e hipertensão essencial. In M. F. P. Oliveira \& S. M. C. Ismael (Orgs.), Rumos da psicologia hospitalar em cardiologia (pp.175-184). Campinas: Papirus.

Lipp, M. E. N. (2000). Manual de aplicação do inventário de sintomas de stress para adultos de Lipp. São Paulo: Casa do Psicólogo.

Lipp, M. E. N., \& Frare, A., Santos, F. U. (2007). Efeitos de variáveis psicológicas na reatividade cardiovascular em momentos de stress emocional. Estudos de Psicologia (Campinas), 24 (2). Recuperado em fevereiro 23, 2008, disponível em http://www.scielo.br/Estpsi

Lipp, M. E. N., Pereira, M. M. B., Justo, A. P., \& Matos, T. M. G. (2006). Cardiovascular reactivity in hypertensives: differential effect of expressing and inhibiting emotions during moments of interpersonal stress. Spanish Journal of Psychology, 9 (2). Retrieved May 4, 2007, available from http://www.ucm.es/info/Psi/docs/journal/ v9_n2_2006//art154.pdf

Lipp, M. E. N., \& Rocha, J. C. (1994). Stress, hipertensão arterial e qualidade de vida. Campinas: Papirus.

Lipp, M. E. N., \& Rocha, J. C. (2007). Pressão alta e stress: o que fazer agora? Um guia de vida para o hipertenso. Campinas: Papirus.

Lipp, M. E. N., \& Tanganelli, M. S. (2002). Stress e qualidade de vida em magistrados da justiça do trabalho: diferenças entre homens e mulheres. Psicologia Reflexão \& Crítica, 15 (3). Recuperado em janeiro 8, 2007, disponível em http:/ /www.scielo.br/pdf/prc/v15n3/a08v15n3.pdf

Lundberg U., \& Frankenhaeuser, M. (1999). Stress and workload in men and women in high-ranking positions. Journal of Occupational Health Psychology, 4 (2). Retrieved May 28, 2007, available from http://gateway.ut.ovid.com

Maciel, C. L. C. (2002). Emoção, doença e cultura: o caso da hipertensão essencial. In B. W. Romano (Org.), A prática da psicologia nos hospitais (pp.2-38). São Paulo: Pioneira Thomson Learning.

Moreira, M. D., \& Mello Filho, J. (1992). Psicoimunologia hoje. In M. F. Júlio (Ed.), Psicossomática hoje (pp.119-151). Porto Alegre: Artes Médicas Sul.

Ribeiro A. B. (1996). Conceito, determinação e classificação da hipertensão arterial. In A. B. Ribeiro (Org.), Atualização em hipertensão arterial: clínica diagnóstico e terapêutica (pp.1-7). São Paulo: Atheneu.

Romano, B. W. (2001). Aspectos psicológicos na determinação do comportamento dos pacientes diante das doenças crônicas. In B. W. Romano (Org.), Psicologia e cardiologia: encontros possíveis (pp.207-221). São Paulo: Casa do Psicólogo.

Schraiber, L. B., Gomes, R., \& Couto, M. T. (2005). Homens e saúde na pauta da saúde Coletiva. Ciência\& Saúde Coletiva, 10 (1). Recuperado em março 1, 2010, disponível em http://www.scielosp.org/pdf/csc/v10n1/a02v10n1.pdf

Selye, H. (1965). Stress: a tensão da vida. São Paulo: IBRASA.

Silva, E. A. T. (2005). Stress e suas repercussões no organismo. In S. M. C. Ismael (Org.), Temas de prevenção, ensino e pesquisa que permeiam o contexto hospitalar (pp.45-54). São Paulo: Casa do Psicólogo.

Sociedade Brasileira de Cardiologia. (1998). III Consenso Brasileiro de Hipertensão Arterial. São Paulo. Recuperado em junho 15, 2006, disponível em http://www.sbh.org. br/documentos/consenso3_documento.htm

Sociedade Brasileira de Cardiologia. (2006). V Diretrizes Brasileiras de Controle da Hipertensão Arterial. São Paulo: SP. Recuperado em janeiro 8, 2007, disponível em http:// www.diabetes.org.br/educacao/documentos/ documents/V_DBHA_2006.pdf

Straub, R. O. (2005). Psicologia da saúde. Porto Alegre: Artmed.

Swales, J. D. (1994). Textbook of hypertension Blackwell. London: Scientific Publications.

Recebido em: 27/1/2009

Versão final reapresentada em: 16/3/2010

Aprovado em: 29/10/2010 